\title{
Content Uniformity in Tablets Evaluated by Energy Dispersive X-ray Spectroscopy
}

\author{
J. Wolfe, T.L. Lersch, and K.L. Bunker
}

RJ Lee Group, Inc., Monroeville, PA 15416

Content uniformity is a vastly important attribute of solid dose pharmaceuticals. The assurance that a constant dose of the product is maintained between tablets is of the utmost importance to ensure patient safety and product efficacy. Traditionally content uniformity is tested via assay. However, the assay technique is bulk in nature and fails to provide information regarding the actual distribution of the ingredients within a given tablet. It would fail, for example, to detect that all of the active ingredient is in one half of a tablet and was not dispersed accurately. This situation could lead to improper dosage for a patient who is splitting pills to achieve the proper dosage. Advanced methods for content uniformity testing now involve product imaging over assay techniques.

In this study, scanning electron microscopy (SEM) with energy dispersive X-ray spectroscopy (EDS) was used to characterize the morphology and content uniformity of a pharmaceutical tablet.

An over-the-counter controlled release medication was examined in a Hitachi S-5500 high resolution SEM. The sample was cleaved and mounted onto a SEM stub. Secondary electron images acquired at $2 \mathrm{kV}$ showing the morphology of the cleaved surface of the tablet are shown in Figure 1. In addition, elemental EDS maps were acquired at $5 \mathrm{kV}$, as shown in Figure 2. The distribution of carbon, aluminum, silicon and magnesium are shown and indicate an inhomogeneity of the components at this magnification.

The highlighted example illustrates how elemental mapping can assist in content uniformity investigations. The technique also exhibits versatility in the ability to analyze different solid dosage forms and also controlled and sustained release systems. For the latter, uniformity of layers in a controlled release system can be evaluated as well as potential layer bleed. Chemical interactions between layers or from spray deposition techniques can also be studied as other examples. To further add to the versatility, the method can also be employed in different phases of the manufacturing process to confirm that the formulation is acting as expected. A range of magnifications can be utilized from whole tablet mapping to magnifications approaching the nano scale depending on the formulation. 

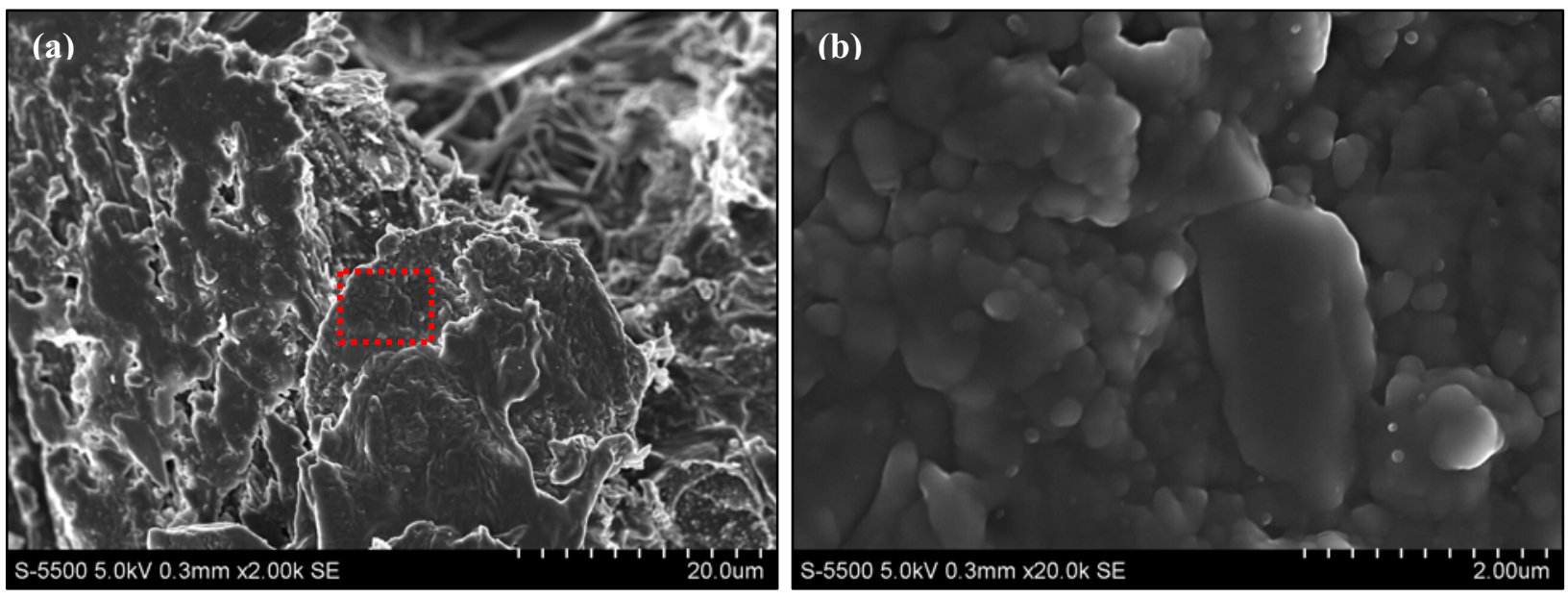

Fig. 1: A (a) $2 \mathrm{kX}$ and (b) $20 \mathrm{kX}$ secondary electron image of a cleaved portion of an over-the-counter pharmaceutical tablet.
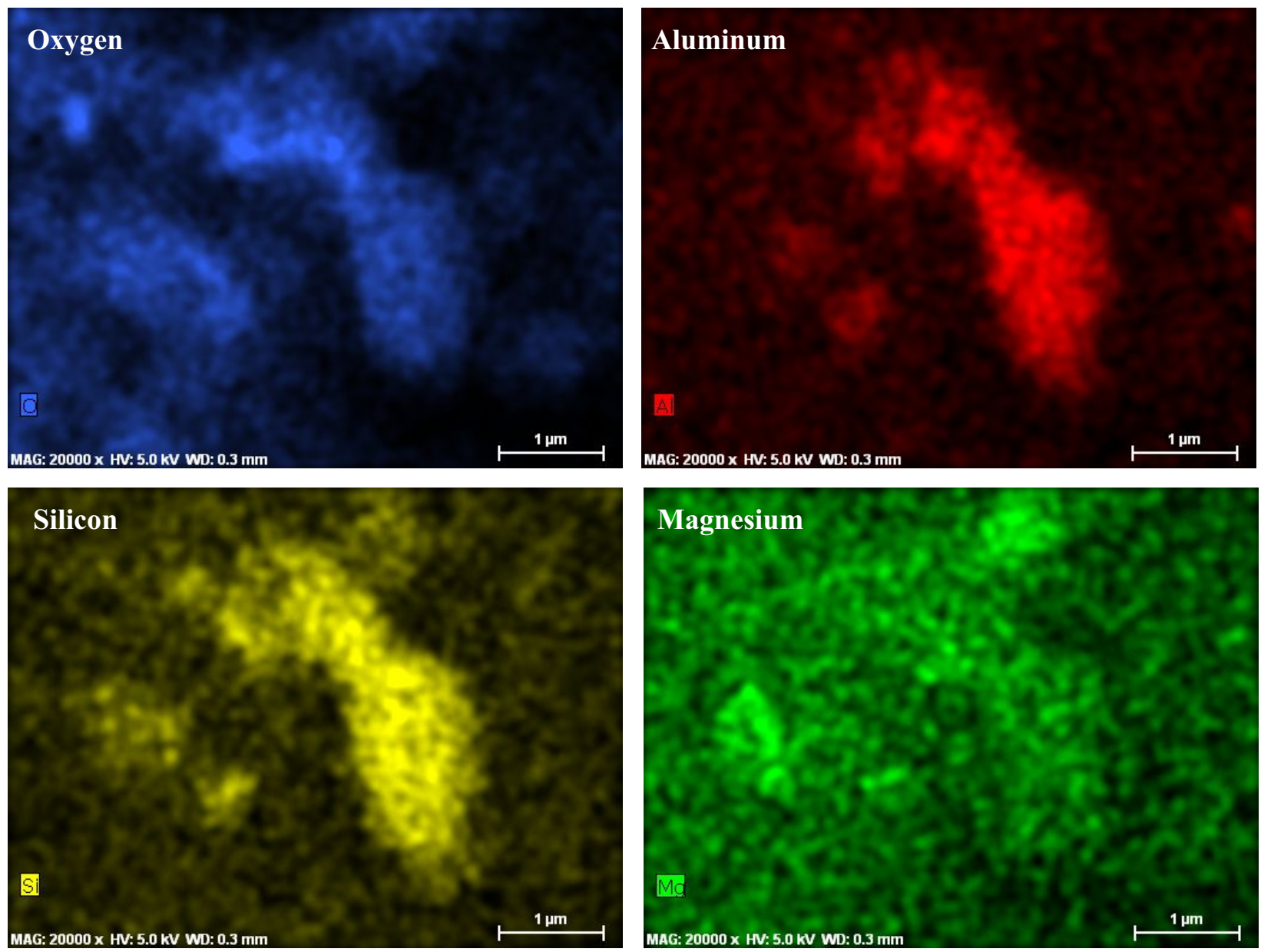

Fig. 2: Elemental EDS maps showing the inhomogeneous distributions of oxygen, aluminum, silicon, and magnesium within the pharmaceutical tablet. The EDS maps were acquired from the area shown in Figure 1 (b). 